\title{
EXISTENCE THEOREMS FOR PARAMETRIC PROBLEMS IN THE CALCULUS OF VARIATIONS AND APPROXIMATION
}

\author{
BY
}

ROBERT M. GOOR

\begin{abstract}
In this paper, we investigate the parametric growth condition which arises in connection with existence theorems for parametric problems of the calculus of variations. In particular, we study conditions under which the length of a curve is dominated in a suitable sense by its "cost". We show that we may restrict our attention to local growth conditions on a particular set. Then we link the growth conditions to a certain approximation problem on this set. Finally, we prove that under suitable topological restrictions related to dimension theory, the local and global problems can be solved.
\end{abstract}

Introduction. In this paper, we prove existence theorems for parametric problems of the calculus of variations with constraints in the form of an orientor field. Our primary interest lies in the parametric growth condition, which concerns the length of admissible trajectories. Our researches along this line have culminated in a single principle which simultaneously unifies and generalizes several diverse classical results of Tonelli: if it is possible to solve a certain approximation problem on a particular distinguished subset, the so called "zero set", of the "space constraint" set, then a satisfactory parametric growth condition is achieved. In $\$ 1$, we define our problem and state a general existence theorem incorporating the growth condition of interest. In $\$ 2$, we prove that we may analytically restrict our attention to the "zero set", and we develop a specific tool for utilizing this fact, thus laying the groundwork for the introduction of the approximation problem in $\S 3$. Known solutions to the approximation problem are cited from another source.

Our formulation of parametric problems in terms of orientor fields is particularly convenient since it includes parametric problems of optimal control, and the existence theory takes its starting point form Tonelli's classical theory. For nonparametric problems, the analogous point of view has been developed by Cesari [1], [2], some of whose results are used here.

1. We assume as given: a set $A$ in the Euclidean space $E^{n}$, for some $n$; a set $B$ in $A \times A$; and, for each $x$ in $A$, a set $Q(x)$ in $E^{n}$. We assume further that

Received by the editors November 19, 1974 and, in revised form, July 25, 1975. AMS (MOS) subject classifications (1970). Primary 49A10, 49A15, 49A30.

Key words and phrases. Parametric problem, calculus of variations, orientor field, growth condition, Fréchet curve, parametric problems of optimal control. 
$Q(x)$ is a cone for every $x$, that is, $k x^{\prime} \in Q(x)$ for every $k \geqslant 0$ and $x^{\prime} \in Q(x)$. Let $M$ be the subset of $E^{2 n}$ defined by

$$
M=\left\{\left(x, x^{\prime}\right) \mid x \in A, x^{\prime} \in Q(x)\right\} .
$$

If $x \in E^{n}$, then $|x|$ will denote the usual Euclidean norm of $x$. For subsets $K, K^{\prime}$ of $E^{n}$, we will use the following notations: $\operatorname{cl} K$ will denote the closure of $K ; K \backslash K^{\prime}$, the set $\left\{x \in K \mid x \notin K^{\prime}\right\}$; and co $K$, the convex hull of $K$.

We assume as given a continuous function $f_{0}$ mapping $M$ into $E^{1}$ such that $f_{0}\left(x, k x^{\prime}\right)=k f_{0}\left(x, x^{\prime}\right)$ for all $x$ in $A, x^{\prime}$ in $Q(x)$ and $k \geqslant 0$. Let $x=x(t), a<t$ $<b$, be a function defined on $[a, b]$ for some $a$ and $b$, with values in $E^{n}$. We will say that $x$ is admissible on $[a, b]$ if:

(1) $x$ is absolutely continuous;

(2) $x(t) \in A$ for all $t$ in $[a, b]$;

(3) $(x(a), x(b)) \in B$;

(4) $x^{\prime}(t) \in Q(x(t))$ for almost all $t$ in $[a, b]$;

(5) $f_{0}\left(x(t), x^{\prime}(t)\right)$ is $L$-integrable on $[a, b]$.

We let $\Omega$ be the class of functions admissible on some compact interval.

If $x=x(t), a \leqslant t \leqslant b$, is an element of $\Omega$, the length of $x$ is $L(x)=$ $\int_{a}^{b}\left|x^{\prime}(t)\right| d t$. It is well known that the length functional is a parametric functional. (See [3, p. 495], for example.)

If $x=x(t), a \leqslant t \leqslant b$, is in $\Omega$, we define the functional $I[x]$ by $I[x]=$ $\int_{a}^{b} f_{0}\left(x(t), x^{\prime}(t)\right) d t$. The proof that the constraints (1)-(5) and functional $I[\cdot]$ give rise to a parametric problem is straightforward, and those details we feel are not reported elsewhere are given in Appendix 1.

We seek conditions on $A, B, Q(\cdot)$ and $f_{0}$ under which $I[\cdot]$ attains its absolute minimum on $\Omega$.

We will say that the set-valued function $Q(x)$ satisfies property $(\mathrm{Q})$ at the point $x$ in $A$ if

$$
Q(x)=\bigcap_{\delta>0} \operatorname{cl} \operatorname{co} \underset{y \in N_{\delta}(x) \cap A}{\bigcup} Q(y),
$$

where $N_{\delta}(x)=\{y|| x-y \mid<\delta\}$. The concept of property (Q) was introduced by Cesari [1] for nonparametric control problems with unbounded control spaces.

For each $x$ in $A$, let $Q_{1}(x)=Q(x) \cap\left\{y \in E^{n}|| y \mid \leqslant 1\right\}$.

THEOREM 1.1. Suppose that: $A$ is compact; $B$ is closed; $Q_{1}(x)$ satisfies property $(Q)$ for every $x$ in $A ; f_{0}\left(x, x^{\prime}\right) \geqslant 0$ for every $\left(x, x^{\prime}\right) \in M$; there exists a continuous function $\Phi: E^{1} \rightarrow E^{1}$ such that $L(x) \leqslant \Phi(I[x])$ for all $x \in \Omega$; and $f_{0}\left(x, x^{\prime}\right)$ is convex in the variable $x^{\prime}$ for each $x \in A$. Then, if $\Omega$ is nonempty, $I[\cdot]$ attains its absolute minimum. 
The proof, given in Appendix II, is a standard application of classical direct methods combined with relatively recent results on lower semicontinuity. We note that the assumption of property $(Q)$ for the sets $Q_{1}(x)$ does not imply the same property for the sets $Q(x)$. For example, if $n=3, A=\left\{\left(x_{1}, x_{2}, x_{3}\right) \mid\right.$ $\left.x_{1}^{2}+x_{2}^{2}+x_{3}^{2}=1\right\}$ and $Q\left(x_{1}, x_{2}, x_{3}\right)$ is the plane $\left\{(x, y, z) \mid x_{1} x+x_{2} y+x_{3} z\right.$ $=0$ \} through the origin parallel to the tangent plane to $A$ at the point $\left(x_{1}, x_{2}, x_{3}\right)$, it is clear that the sets $Q_{1}\left(x_{1}, x_{2}, x_{3}\right)$ satisfy property $(Q)$ while the sets $Q\left(x_{1}, x_{2}, x_{3}\right)$ do not. For more information on property $(Q)$ as it relates to the sets $Q(x)$ and $Q_{1}(x)$, see Kaiser [9].

The considerations above and Theorem 1.1 apply to parametric Lagrange problems of optimal control. Indeed, suppose that

$$
I[x, u]=\int_{a}^{b} f_{0}(t, x(t), u(t)) d t,
$$

subject to

$$
d x / d t=f(t, x(t), u(t)), \quad t \in[a, b](\text { a.e. }),
$$

$$
x(t) \in A \subset E^{n}, u(t) \in U(t, x(t)) \subset E^{m}, \quad(x(a), x(b)) \in B \subset A \times A,
$$

for some given continuous functions $f_{0}, f$, sets $A, U(t, x)$ and integers $n, m$. Letting $\tilde{f}=\left(f_{0}, f\right), \widetilde{Q}(t, x)=\tilde{f}(t, x, U(t, x))$ and $\tilde{x}=\left(x^{0}, x\right)$, we may rephrase the given Lagrange problem in the following equivalent form:

$$
I[\widetilde{x}]=\int_{a}^{b}\left(d x^{0} / d t\right) d t, d \widetilde{x} / d t \in \widetilde{Q}(t, x(t)), \widetilde{x}(t) \in E^{1} \times A,
$$

$$
(x(a), x(b)) \in B, \quad t \in[a, b] \text { (a.e.), with } I[\widetilde{x}]=I[x, u]
$$

If $\widetilde{Q}(t, x)$ happens to be $t$-independent and a cone for each $x$ in $A$, the theory underlying Theorem 1.1 may apply directly, thus giving an existence result for the original Lagrange problem of optimal control. Problems (1), (2), with the provision that $\widetilde{Q}(t, x)$ be $t$-independent and a cone, will be denoted as parametric Lagrange problems. We shall prove in Appendix 1 that they are indeed parametric, that is, independent of the "representation", and we shall be specific on the concept of representation. Meanwhile, we note here that, if an absolutely continuous function $\tilde{x}(t), a \leqslant t \leqslant b$, satisfies (3), then a corresponding measurable control function $u(t)$ for which (1), (2) hold and $I[\tilde{x}]=I[x, u]$ is known to exist by force of the McShane-Warfield theorem [11]. In any case, however, we may define $\bar{x}=\left(t, x^{0}, x\right)$ and $R(\bar{x})=\{1\} \times \widetilde{Q}(t, x)$ so that

$$
I[x, u]=I[\bar{x}]=\int_{a}^{b}\left(d x^{0} / d \tau\right) d \tau,
$$

subject to

$$
\begin{gathered}
d \bar{x} / d \tau \in R(\bar{x}(\tau)), \\
\bar{x}(\tau) \in E^{2} \times A, \quad(\bar{x}(a), \bar{x}(b)) \in\{a\} \times\{0\} \times B \times\{b\} \times E^{1} .
\end{gathered}
$$


Here, however, the set-valued function $R(\bar{x})$ is not a cone for any $\bar{x}$. Let $S(\bar{x})$ $=\{k r \mid r \in R(\bar{x}), k \geqslant 0\}$. Then, $S(\bar{x})$ is a cone and the problem (P2) of minimizing $I[\bar{x}]$ subject to $d \bar{x} / d \tau \in S(\bar{x}(\tau))$ is a parametric problem. Furthermore, it is easy to see that if (P2) has an absolute minimum (with $t(\tau)$ constant on no interval), so does the functional (P1), and hence the original Lagrange functional has an absolute minimum. However, the applicability of Theorem 1.1 to problems in this form is limited by the requirement that the sets $S_{1}(\bar{x})=S(\bar{x}) \cap$ $\left\{q \in E^{n+2}|| q \mid \leqslant 1\right\}$ must satisfy property (Q) for each $\bar{x}$. This geometric condition underlies a growth condition on $f_{0}$ and $f$ distinct from that imposed on the length $L(x)$ in Theorem 1.1. The condition $L(x) \leqslant \Phi(I[x])$ was used by Tonelli [13] in his existence analysis for the free parametric problems of calculus of variations, and used later also by McShane [10].

In this paper, our interest lies with the growth condition used in Theorem 1.1. We will say that $I \Phi$-dominates $L$ for some $\Phi$ if there is a continuous function $\Phi: E^{1} \rightarrow E^{1}$ such that $L(x) \leqslant \Phi(I[x])$ for all $x \in \Omega$. We seek conditions on $A, Q(x)$ and $f_{0}$ under which $I \Phi$-dominates $L$ for some function $\Phi$.

We note that if $G(x)$ is any continuously differentiable real-valued function defined in a neighborhood of the compact set $A$, with gradient $\nabla G(x)$, then the function

$$
F_{0}\left(x, x^{\prime}\right)=f_{0}\left(x, x^{\prime}\right)+\nabla G(x) \cdot x^{\prime}
$$

when regarded as the integrand of a parametric functional, gives rise to the same $\Phi$-dominance properties as the function $f_{0}$. That is, if $J[x]=\int_{a}^{b} F_{0}\left(x(t), x^{\prime}(t)\right) d t$, it is clear that $I \Phi$-dominates $L$ for some $\Phi$ if and only if $J \Psi$-dominates $L$ for some $\Psi$. Furthermore, $I[\cdot]$ is lower semicontinuous if and only if $J[\cdot]$ is. Consequently, the condition $f_{0}\left(x, x^{\prime}\right) \geqslant 0$ in Theorem 1.1 may be replaced by the condition

$$
f_{0}\left(x, x^{\prime}\right)+\nabla G(x) \cdot x^{\prime} \geqslant 0
$$

for some function $G$, as above.

In accordance with Theorem 1.1, we will assume for the remainder of this paper that $A$ is compact, $M$ is closed and $f_{0}\left(x, x^{\prime}\right) \geqslant 0$ for all $\left(x, x^{\prime}\right) \in M$.

2. All of the known results (including those of this paper) on $I \Phi$-dominance depend on the geometric properties of a particular distinguished subset $A_{0}$ of $A$, defined below:

$$
A_{0}=\left\{x \in A \mid f_{0}\left(x, x^{\prime}\right)=0 \text { for some } x^{\prime} \in Q(x), x^{\prime} \neq 0\right\} .
$$

It is trivial that $A_{0}$ is compact. 
THEOREM 2.1. If $A_{0}$ is empty, there is a fixed number $\nu>0$ such that $\nu\left|x^{\prime}\right| \leqslant f_{0}\left(x, x^{\prime}\right)$ for all $x^{\prime} \in Q(x)$ and all $x \in A$.

Corollary 2.1. If $A_{0}$ is empty, then $L(x) \leqslant(1 / v) I[x]$ for all $x$ in $\Omega$ (with $\nu$ given by Theorem 2.1).

The proofs of these results essentially duplicate Tonelli's theorems for free parametric problems [13, Volume II, p. 10, 2 , No. 6.1], and are omitted. See also [6].

We are interested in the case $A_{0} \neq \varnothing$. Our first aim is to show that we can restrict our attention to $A_{0}$ in our study of $\Phi$-dominance.

Suppose that $x=x(t), a \leqslant t \leqslant b$, is an absolutely continuous function such that $x(t) \in A$ for $a \leqslant t \leqslant b$. If $W$ is an open subset of $E^{n}$ and if $\bar{x}=x(t)$, $c \leqslant t \leqslant d$, is a subarc of $x$ such that $x(t) \in W$ for $c<t<d$, we will call $\bar{x}$ a $W$-subarc of $x$. We will say that $I \Psi$-dominates $L$ locally if there exists an open neighborhood $W$ of $A_{0}$ and a continuous function $\Psi$ such that $L(\bar{x}) \leqslant \Psi(I[x])$ for every $x$ in $\Omega$ and every $W$-subarc $\bar{x}$ of $x$. The following result reduces the problem of $I \Phi$-dominance to one of $I \Psi$-dominance locally.

THEOREM 2.2. If I $\Psi$-dominates $L$ locally for some $\Psi$, then $I \Phi$-dominates $L$ for some $\Phi$.

Proof. Let $W$ be the neighborhood of $A_{0}$ guaranteed by the hypothesis. Let $X$ and $Y$ be open neighborhoods of $A_{0}$ such that $Y \subseteq \operatorname{cl} Y \subseteq X \subseteq \operatorname{cl} X \subseteq W$. Let $\Psi$ be a continuous function, $\Psi: E^{1} \rightarrow E^{1}$, such that $L(\bar{x}) \leqslant \Psi(I[x])$ for all $x \in \Omega$ and for all $W$-subarcs $\bar{x}$ of $x$. Let $\delta$ be the distance from $\partial X$ to $\partial Y$. Since $\operatorname{cl} Y \subseteq X$, we know that $\delta$ is positive.

We let $D_{1}$ be the set of all functions $x$ admissible on some interval $[a, b]$, such that $x(t) \in \operatorname{cl} X$ for $a \leqslant t \leqslant b$; we let $D_{2}$ be the set of all functions $x$ admissible on some interval $[a, b]$, such that $x(t) \in A \backslash Y$ for $a \leqslant t \leqslant b$; and we let $D_{3}=\Omega \backslash\left(D_{1} \cup D_{2}\right)$.

By assumption,

$$
L(x) \leqslant \Psi(I[x])
$$

for all $x$ in $D_{1}$.

Since $Y$ is an open neighborhood of the zero set $A_{0}, A \backslash Y$ is compact. Therefore, since $f_{0}\left(x, x^{\prime}\right)>0$ for $x^{\prime} \neq 0, x \in A \backslash Y$, it follows from Theorem 2.1 that there is a fixed $\nu^{\prime}>0$ such that

$$
f_{0}\left(x, x^{\prime}\right) \geqslant \nu^{\prime}\left|x^{\prime}\right|
$$

for all $x$ in $A \backslash Y, x^{\prime} \in Q(x)$. By Corollary 2.1, 
for all $x \in D_{2}$.

We now consider any element $x$ of $D_{3}$, where $x$ is admissible on $[a, b]$. Since $x \notin D_{1} \cup D_{2}$, the trajectory $x(t)$ includes points of $Y$ as well as points of $A \backslash X$. We let $E=x^{-1}(X)$ and $F=x^{-1}(A \backslash Y)$. Since $X$ is open and $x=x(t)$ is continuous, $E$ is relatively open with respect to $[a, b]$. Therefore, $E$ is the union of countably many disjoint nonempty relatively open intervals $I^{k}, k=1,2, \ldots$ Suppose that for some $k, x\left(\mathrm{cl} I^{k}\right) \cap \partial X$ is empty. Then $\mathrm{cl} I^{k}$ is a subset of $E$, which implies that $I^{k}=\mathrm{cl} I^{k}$. This can happen only if $I^{k}=[a, b]$. Since this would imply that $x \in D_{1}$, we conclude that $x\left(\operatorname{cl} I^{k}\right) \cap \partial X$ is nonempty for each $k=1,2, \ldots$.

We consider only those intervals $B_{j}=I^{k_{j}}, j=1,2, \ldots$, for which $x\left(B_{j}\right)$ $\cap Y$ is nonempty, and hence, $x\left(B_{j}\right) \cap \partial Y$ is nonempty. For $j=1,2, \ldots$, we define the absolutely continuous function $x_{j}=x_{j}(t), t \in \mathrm{cl} B_{j}$, by $x_{j}(t)=x(t)$ for $t$ in $\operatorname{cl} B_{j}$. By the definition of $\delta, L\left(x_{j}\right) \geqslant \delta$ for each $j$. Also, $\Sigma_{j=1}^{\infty} L\left(x_{j}\right) \leqslant$ $L(x)$, which is finite. It follows that there may be only finitely many of the intervals $B_{j}$, since each length $L\left(x_{j}\right)$ is bounded below by $\delta$. Let $N$ be the number of intervals $B_{j}$. Let $E_{j}$ be a closed subinterval of $\operatorname{cl} B_{j}$ such that $x\left(E_{j}\right) \subseteq$ $\operatorname{cl} X \backslash Y$ and such that the arc $x\left(E_{j}\right)$ joins $\partial X$ and $\partial Y$. Let $L_{j}$ be the length of $x$ restricted to $E_{j}$, so that $L_{j}=\int_{E_{j}}\left|x^{\prime}(t)\right| d t$. Let $L_{F}=\int_{F}\left|x^{\prime}(t)\right| d t$. It follows that $L_{j} \geqslant \delta$ for $j=1,2, \ldots, N$, and $\Sigma_{j=1}^{N} L_{j} \geqslant N \delta$. But, $x\left(E_{j}\right) \subseteq \mathrm{cl} X \backslash Y$ for each $j$ implies that $\bigcup_{j=1}^{N} E_{j} \subseteq F$ and therefore, writing $G=\bigcup_{j=1}^{N} E_{j}$,

Thus,

$$
\sum_{j=1}^{N} L_{j}=\int_{G}\left|x^{\prime}(t)\right| d t<\int_{F}\left|x^{\prime}(t)\right| d t=L_{F}
$$

$$
N \leqslant(1 / \delta) L_{F}
$$

But, by (2), $\left(1 / \nu^{\prime}\right) f_{0}\left(x, x^{\prime}\right) \geqslant\left|x^{\prime}\right|$ for all $x$ in $A \backslash Y, x^{\prime}$ in $Q(x)$, so that

$$
L_{F} \leqslant\left(1 / \nu^{\prime}\right) \int_{F} f_{0}\left(x(t), x^{\prime}(t)\right) d t .
$$

Since $f_{0}\left(x, x^{\prime}\right) \geqslant 0$ for all $\left(x, x^{\prime}\right) \in M$,

$$
L_{F} \leqslant\left(1 / v^{\prime}\right) I[x],
$$

so, by (4) we have

$$
N<\left(1 / \delta v^{\prime}\right) I[x] .
$$

Now, $B_{j}$ is an interval such that $x\left(B_{j}\right) \subseteq \mathrm{cl} X$ so, by assumption, $L\left(x_{j}\right) \leqslant$ $\Psi(I[x])$. In light of (6), we have

$$
\sum_{j=1}^{N} L\left(x_{j}\right) \leqslant N \Psi(I[x]) \leqslant\left(1 / \delta \nu^{\prime}\right) I[x] \Psi(I[x]) .
$$


Let $F^{\prime}=[a, b] \backslash F=x^{-1}(Y)$. Since $x(t) \in Y$ implies $t$ is in $B_{j}$ for some $j, 1<j \leqslant N, F^{\prime} \subseteq \bigcup_{j=1}^{N} B_{j}$. Therefore,

and we have that

$$
L(x)=\int_{F}\left|x^{\prime}(t)\right| d t+\int_{F^{\prime}}\left|x^{\prime}(t)\right| d t
$$

Combining (5) and (7), we have

$$
L(x) \leqslant L_{F}+\sum_{j=1}^{N} L\left(x_{j}\right)
$$

$$
L(x) \leqslant\left(1 / \delta \nu^{\prime}\right) I[x] \Psi(I[x])+\left(1 / \nu^{\prime}\right) I[x] .
$$

Since $\delta, \nu^{\prime}, \Psi$ are fixed independently of the particular element $x$ of $D_{3}$, relation (8) holds for all $x \in D_{3}$.

For $-\infty<\alpha<\infty$ we define $\Phi(\alpha)=\max \left\{\Psi(\alpha), \alpha / \nu^{\prime},\left(\alpha / \delta \nu^{\prime}\right) \Psi(\alpha)+\left(\alpha / \nu^{\prime}\right)\right\}$. By the continuity of the three functions in brackets, $\Phi$ is continuous on $E^{1}$, and, by formulas (1), (3) and (8), $L(x) \leqslant \Phi(I[x])$ for all $x$ in $\Omega$. That is, $I$ $\Phi$-dominates $L$.

We conclude this section with a theorem which will be our primary tool for exploiting Theorem 2.2 .

THEOREM 2.3. Suppose there is $a \nu>0$, and a continuously differentiable real-valued function $G$ defined on a neighborhood $W$ of $A_{0}$ having gradient $\nabla G$, such that $f_{0}\left(x, x^{\prime}\right) \geqslant \nabla G(x) \cdot x^{\prime}+\nu\left|x^{\prime}\right|$ for all $x \in A \cap W$ and $x^{\prime} \in Q(x)$. Then $I \Phi$-dominates $L$ for some $\Phi$.

Proof. We will show that $I \Psi$-dominates $L$ locally. Let $X$ be any neighborhood of $A_{0}$ such that $\mathrm{cl} X \subseteq W$. Then by hypothesis,

$$
f_{0}\left(x, x^{\prime}\right) \geqslant \nabla G(x) \cdot x^{\prime}+\nu\left|x^{\prime}\right|
$$

for all $x$ in $A \cap \mathrm{cl} X$ and $x^{\prime}$ in $Q(x)$. Let $x$ be an admissible function on the interval $[a, b]$ and let $\bar{x}=x(t), c \leqslant t \leqslant d$, be any $X$-subarc of $x$. Then,

$$
f_{0}\left(x(t), x^{\prime}(t)\right) \geqslant \nabla G(x(t)) \cdot x^{\prime}(t)+\nu\left|x^{\prime}(t)\right|
$$

for $c<t<d$. We integrate over $(c, d)$ and use the fact that $f_{0}\left(x, x^{\prime}\right) \geqslant 0$ to obtain

$$
I[x] \geqslant G(x(d))-G(x(c))+\nu L(\bar{x}) .
$$

Let $\alpha=1 / \nu$ and $\beta=(2 v) \max |G(x)|$, where the maximum is taken for $x$ in cl $X$. Then, $L(\bar{x}) \leqslant \alpha I[x]+\beta$. It follows that $I \Psi$-dominates $L$ locally, where $\Psi(t)=$ $\alpha t+\beta$. By Theorem 2.2,I $\Phi$-dominates $L$ for some $\Phi$.

We illustrate Theorem 2.3 with the following example. Suppose $n=2$, $A=\left\{(x, y) \mid x^{2}+y^{2}<9\right\}, Q(x, y)=E^{2}$ for all $(x, y) \in A$, and $f_{0}(x, y, u, v)=$ $\left(u^{2}+v^{2}\right)^{3 / 2}\left(\left[u\left(u^{2}+v^{2}\right)^{-1 / 2}-x\right]^{2}+\left[v\left(u^{2}+v^{2}\right)^{-1 / 2}-y\right]^{2}\right)^{1 / 2}$. Then, it is clear 
that $A_{0}=\left\{(x, y) \mid x^{2}+y^{2}=1\right\}$. For the present, we restrict consideration to the case $\left(x^{\prime}\right)^{2}+\left(y^{\prime}\right)^{2}=x^{2}+y^{2}=1$. Let $\bar{x}=(x, y)$ and $\bar{u}=\left(x^{\prime}, y^{\prime}\right)$, so that $|\bar{x}|=|\bar{u}|=1$. Then $|\bar{x}-\bar{u}|^{2}=2-2 \bar{x} \cdot \bar{u}$. But $|\bar{x}-\bar{u}| \leqslant 2$ implies that $|\bar{x}-\bar{u}|^{2}$ $\leqslant 2|\bar{x}-\bar{u}|$, and combining this inequality with the expression for $|\bar{x}-\bar{u}|^{2}$ gives $|\bar{x}-\bar{u}| \geqslant 1-\bar{x} \cdot \bar{u}$. Therefore,

$$
|\bar{x}-\bar{u}|>1 / 2-\bar{x} \cdot \bar{u}
$$

for $|\bar{x}|=|\bar{u}|=1$. Let

$$
g(x, y, u, v)=1 / 2\left(u^{2}+v^{2}\right)^{1 / 2}-(x u+y v)
$$

for all $(x, y, u, v)$ in $E^{4}$. Then, we have shown that

$$
g(x, y, u, v)<f_{0}(x, y, u, v)
$$

for all $(x, y, u, v)$ such that $x^{2}+y^{2}=u^{2}+v^{2}=1$. It follows that there is an open neighborhood $W$ of $A_{0}$ such that $g(x, y, u, v) \leqslant f_{0}(x, y, u, v)$ for $(x, y)$ in $W, u^{2}+v^{2}=1$. By the homogeneity of $g$ and $f_{0}$, this yields that $g(x, y, u, v)$ $\leqslant f_{0}(x, y, u, v)$ for all $(x, y)$ in $W,(u, v)$ in $E^{2}$. We let $G(x, y)=-\left(x^{2}+y^{2}\right) / 2$. We have thus shown that

$$
\nabla G(x, y) \cdot\left(x^{\prime}, y^{\prime}\right)+1 / 2\left|\left(x^{\prime}, y^{\prime}\right)\right| \leqslant f_{0}\left(x, y, x^{\prime}, y^{\prime}\right)
$$

for all $(x, y)$ in $W$ and $\left(x^{\prime}, y^{\prime}\right)$ in $E^{2}$. If $I[\cdot]$ is the functional associated with $f_{0}$, then by Theorem 2.3, $I \Phi$-dominates $L$ for some $\Phi$.

REMARK. It is well known (see Tonelli [13], for example) that if

$$
f_{0}\left(x, x^{\prime}\right) \geqslant \nabla G(x) \cdot x^{\prime}+\nu\left|x^{\prime}\right|
$$

for all $\left(x, x^{\prime}\right)$ in $M$, for some fixed $\nu>0$ and some continuously differentiable function $G(x)$, then $I \Phi$-dominates $L$ for some $\Phi$. However, as the above example illustrates, Theorem 2.3 shows that an inequality of this type need be verified only in a neighborhood of $A_{0}$.

3. Our first application of Theorem 2.3 is for situations in which no restrictions are placed on $A_{0}$. We define $Q_{0}(x)=\left\{x^{\prime} \in Q(x) \mid f_{0}\left(x, x^{\prime}\right)=0\right\}$ for $x \in A$. Thus, $Q_{0}(x)=\{0\}$ for $x \in A \backslash A_{0}$, and $Q_{0}(x)$ is a nontrivial cone for $x \in A_{0}$.

THEOREM 3.1. Suppose there is a unit vector $b^{\prime}$ in $E^{n}$ such that $b^{\prime} \cdot x^{\prime}$ $<0$ for all $x^{\prime}$ in $Q_{0}(x), x^{\prime} \neq 0$ and all $x \in A_{0}$. Then, there is a fixed $b \in E^{n}$ and $\nu>0$ such that $f_{0}\left(x, x^{\prime}\right) \geqslant b \cdot x^{\prime}+\nu\left|x^{\prime}\right|$ for all $\left(x, x^{\prime}\right) \in M$.

REMARK. An analogue to this result was first proved by Tonelli in [13, Volume II, p. 27] for free parametric problems and for the case $n=2$.

Proof. From the fact that the set $\left\{\left(x, x^{\prime}\right)\left|x \in A_{0}, x^{\prime} \in Q_{0}(x),\right| x^{\prime} \mid=1\right\}$ 
is compact, it is straightforward to show that there is an open set $X$ with the following properties: $Q_{0}(x) \subseteq X \cup\{0\}$ for all $x$ in $A, \operatorname{cl} X$ is a cone and there is a fixed constant $c_{1}>0$ so that

$$
b^{\prime} \cdot x^{\prime} \leqslant-c_{1}\left|x^{\prime}\right|
$$

for all $x^{\prime}$ in $\operatorname{cl} X$. We define the set $Q^{\prime}(x)=\left\{x^{\prime} \in Q(x) \mid x^{\prime} \notin X\right\}$ and we let $\bar{M}=\left\{\left(x, x^{\prime}\right) \mid x \in A, x^{\prime} \in Q^{\prime}(x)\right\}$. It is clear that $\bar{M}$ is closed and $f_{0}\left(x, x^{\prime}\right)>0$ for $\left(x, x^{\prime}\right) \in \bar{M}, x^{\prime} \neq 0$. By Theorem 2.1, there is a constant $c_{2}>0$ such that

$$
f_{0}\left(x, x^{\prime}\right) \geqslant c_{2}\left|x^{\prime}\right|
$$

for $\left(x, x^{\prime}\right)$ in $\bar{M}$. We now rewrite (1) as

$$
\left[c_{1} / c_{2}\right]\left[\left(c_{2} / c_{1}\right) b^{\prime} \cdot x^{\prime}+c_{2}\left|x^{\prime}\right|\right] \leqslant 0 \leqslant f_{0}\left(x, x^{\prime}\right),
$$

for all $x^{\prime}$ in $Q(x) \cap X$. For $x^{\prime}$ in $Q(x) \cap X$, this implies

$$
\left(c_{2} / c_{1}\right) b^{\prime} \cdot x^{\prime}+c_{2}\left|x^{\prime}\right| \leqslant f_{0}\left(x, x^{\prime}\right) .
$$

For $x^{\prime}$ in $Q(x) \backslash X$, we have

$$
\left(c_{2} / c_{1}\right) b^{\prime} \cdot x^{\prime} \leqslant\left|\left(c_{2} / c_{1}\right) b^{\prime} \cdot x^{\prime}\right| \leqslant\left(c_{2} / c_{1}\right)\left|x^{\prime}\right| \leqslant\left(1 / c_{1}\right) f_{0}\left(x, x^{\prime}\right),
$$

so combining (2) and (4), for $\left(x, x^{\prime}\right)$ in $\bar{M}$,

$$
\left(c_{2} / c_{1}\right) b^{\prime} \cdot x^{\prime}+c_{2}\left|x^{\prime}\right| \leqslant\left(1+1 / c_{1}\right) f_{0}\left(x, x^{\prime}\right) .
$$

Since (5) holds for $\left(x, x^{\prime}\right)$ in $M \backslash \bar{M}$ as well, by (3), we have shown (5) for all $\left(x, x^{\prime}\right)$ in $M$. Letting $b=\left[c_{2} /\left(c_{1}+1\right)\right] b^{\prime}$ and $\nu=c_{1} c_{2} /\left(c_{1}+1\right)$, we have that

$$
f_{0}\left(x, x^{\prime}\right) \geqslant b \cdot x^{\prime}+\nu\left|x^{\prime}\right|
$$

for all $\left(x, x^{\prime}\right)$ in $M$.

\section{COROLlaRY 3.1. Under the hypotheses of Theorem 3.1, I $\Phi$-dominates} $L$ for some continuous function $\Phi$.

Proof. Theorem 2.3 applies with $G(x)=b \cdot x$.

We consider an example with $n=4, Q(x)=Q\left(x_{1}, x_{2}, x_{3}, x_{4}\right)=Q=$ $\left\{\left(x_{1}^{\prime}, x_{2}^{\prime}, x_{3}^{\prime}, x_{4}^{\prime}\right) \mid x_{4}^{\prime} \geqslant 0, \Sigma_{i=1}^{3}\left(x_{i}^{\prime}\right)^{2} \leqslant\left(x_{4}^{\prime}\right)^{2}\right\}$. Let $\psi(x)=\left(\psi_{1}(x), \psi_{2}(x), \psi_{3}(x)\right.$, $\left.\psi_{4}(x)\right)$ be a continuous vector function and let $\nu_{1}, \nu_{2}$ be positive constants. We define

$$
f_{0}\left(x, x^{\prime}\right)=\nu_{1} \psi(x) \cdot x^{\prime}-\nu_{2}\left[\left(x_{4}^{\prime}\right)^{2}-\sum_{i=1}^{3}\left(x_{i}^{\prime}\right)^{2}\right]^{1 / 2} .
$$

If $x_{4}$ is identified with $c$ times the "time" coordinate of a particle (where $c$ is the speed of light) and $\left(x_{1}, x_{2}, x_{3}\right)$ are its "space" coordinates, then this form of parametric integrand arises in connection with the variational formulation of the single particle special theory of relativity (see Rund [12, pp. 182-183]). In 
this case, the radical is the (negative of the) "free particle Lagrangian" and the inner product term is the (negative of the) interaction Lagrangian. In case of a particle in an electromagnetic field, the vector function $\psi(x)$ is the 4-potential of the field. It follows that the interesting cases are those for which $\psi(x)$ does not equal $\nabla G(x)$ for some function $G(x)$, for $\psi(x)=\nabla G(x)$ implies there are no forces of interaction (see Rund [12, p. 184]). We observe that for $x$ in a compact set $A$, if $\nu_{3}$ is large enough, then $F_{0}\left(x, x^{\prime}\right)=f_{0}\left(x, x^{\prime}\right)+\nu_{3} x_{4}^{\prime} \geqslant 0$. Let $J[\cdot]$ be the integral functional with integrand $F_{0}: J[x]=\int_{a}^{b} F_{0}\left(x(t), x^{\prime}(t)\right) d t$. Then, since $Q_{0}(x) \subseteq Q$ and $b=(0,0,0,-1)$ satisfies $b \cdot x^{\prime}<0$ for all nonzero $x^{\prime}$ in $Q$, Theorem 3.1 and Corollary 3.1 apply and $J \Psi$-dominates $L$ for some $\Psi$. By the remark at the end of $\S 1$, it follows that $I \Phi$-dominates $L$ for some $\Phi$.

We now study restrictions on the zero set $A_{0}$ which guarantee that $I$ $\Phi$-dominates $L$ for some $\Phi$.

We will say that $f_{0}$ satisfies the local growth condition (*) on the compact set $K \subseteq A$ if, for each $x_{0}$ in $K$, there is a vector $b$ in $E^{n}$ and scalars $\nu>0, \delta>$ 0 such that $f_{0}\left(x, x^{\prime}\right) \geqslant b \cdot x^{\prime}+\nu\left|x^{\prime}\right|$ for all $x$ in $N_{\delta}\left(x_{0}\right) \cap A$ and $x^{\prime}$ in $Q(x)$.

REMARK. This local growth condition is related to concepts of normality and seminormality introduced by Tonelli ([13, Volumes I and II] and [14]) for both parametric and nonparametric free problems of the calculus of variations. These concepts were used by Tonelli, McShane and others, and later reworded by Turner in [15]. An integrand $f_{0}\left(x, x^{\prime}\right)$ for free parametric problems $(Q(x)=$ $E^{n}$ for all $\left.x\right)$ is said to be normal at a point $(\bar{x}, \bar{u}),|\bar{u}|=1$, if given $\epsilon>0$, there are $\delta>0, \nu>0$ and a vector $b$ in $E^{n}$ such that:

(i) $f_{0}\left(x, x^{\prime}\right) \geqslant b \cdot x^{\prime}+\nu\left|x^{\prime}\right|$ for all $x$ in $N_{\delta}(\bar{x}), x^{\prime}$ in $E^{n}$;

(ii) $f_{0}\left(x, x^{\prime}\right) \leqslant b \cdot x^{\prime}+\epsilon\left|x^{\prime}\right|$ for all $x$ in $N_{\delta}(\bar{x}), x^{\prime}$ in $E^{n},\left|\left(x^{\prime} /\left|x^{\prime}\right|\right)-\bar{u}\right|$ $<\delta$.

Thus, we see that local growth condition (*) is similar to part (i) of the concept of normality. A number of rather deep properties of normal integrands were proved by Tonelli ([13] and [14]) and reproved by Turner solely under continuity and convexity assumptions. For extensions of some of these properties and for an analysis of property (Q) as an extension of seminormality for nonparametric control problems, see Cesari [4].

Lemma 3.1. If $f_{0}$ satisfies the local growth condition $(*)$ on $A_{0}$, then there is a continuously differentiable vector field $b(x)$ defined in a neighborhood $W$ of $A_{0}$, and a scalar $\nu>0$ such that

$$
f_{0}\left(x, x^{\prime}\right) \geqslant b(x) \cdot x^{\prime}+\nu\left|x^{\prime}\right|
$$

for all $x$ in $A \cap W$ and $x^{\prime}$ in $Q(x)$.

Proof. By local growth condition (*) and the compactness of $A_{0}$, it is 
clear that there are finitely many elements $x_{1}, \ldots, x_{k}$ of $A_{0}$, vectors $b_{1}$, $\ldots, b_{k}$ in $E^{n}$, scalars $\delta_{1}, \ldots, \delta_{k}$, and $v_{1}, \ldots, v_{k}$, all positive, such that $f_{0}\left(x, x^{\prime}\right) \geqslant b_{j} \cdot x^{\prime}+v_{j}\left|x^{\prime}\right|$ for all $x$ in $B_{j} \cap A, x^{\prime}$ in $Q(x)$, where $B_{j}$ is the open ball of radius $\delta_{j}$ centered at $x_{j}$, and $A_{0} \subseteq B_{1} \cup B_{2} \cup \cdots \cup B_{k}$. Let $\nu=$ $\min \left(\nu_{1}, \ldots, v_{k}\right)>0$. Then,

$$
f_{0}\left(x, x^{\prime}\right) \geqslant b_{j} \cdot x^{\prime}+v\left|x^{\prime}\right|
$$

for each $x$ in $B_{j} \cap A$ and $x^{\prime}$ in $Q(x)$. Suppose $y \in B_{j} \cap A$ for $j$ in an index set $J \subseteq\{1,2, \ldots, k\}$, and let $\left\{\lambda_{j}\right\}_{j \in J}$ be nonnegative numbers such that $\Sigma_{j \in J} \lambda_{j}$ $=1$. Then, for all $x^{\prime}$ in $Q(y)$, it is an easy consequence of (6) that

$$
f_{0}\left(y, x^{\prime}\right) \geqslant\left(\sum_{j \in J} \lambda_{j} b_{j}\right) \cdot x^{\prime}+\nu\left|x^{\prime}\right| .
$$

Let $W$ be an open neighborhood of $A_{0}$ such that $\mathrm{cl} W \subseteq B_{1} \cup \cdots \cup B_{k}$. We let $c_{1}(x), \ldots, c_{k}(x)$ be a continuously differentiable partition of unity for the sets $W \cap B_{j}$ so that $\sum_{j=1}^{k} c_{j}(x)=1$ for $x$ in $\mathrm{cl} W$. Define $b(x)$ by setting $b(x)=$ $\sum_{j=1}^{k} b_{j} c_{j}(x)$. Then $b(x)$ is a continuously differentiable vector field defined in the neighborhood $W$ of $A_{0}$ and, from (7),

$$
f_{0}\left(x, x^{\prime}\right) \geqslant b(x) \cdot x^{\prime}+\nu\left|x^{\prime}\right|
$$

for each $x$ in $W \cap A$, and $x^{\prime}$ in $Q(x)$.

The conclusion of Lemma 3.1 resembles the hypothesis of Theorem 2.3, with one difference. If the vector field $b(x)$ in the lemma could be replaced by $\nabla G(x)$ for some continuously differentiable function $G$, then Theorem 2.3 would apply and we could conclude that $I \Phi$-dominates $L$. Our final goal in this paper is to establish conditions on $A_{0}$ under which local growth condition (*) implies $\Phi$-dominance (via Theorem 2.3). The conditions will concern suitable properties of "slenderness" of $A_{0}$ and related topological properties. We will now show that if a particular approximation problem on $A_{0}$ has a solution, then the local growth condition (*) on $A_{0}$ implies that $I \Phi$-dominates $L$ for some $\Phi$. We require the following definition.

If $b(x)$ is a continuous vector field defined on a compact subset $K$ of $E^{n}$ (i.e., $b(x) \in E^{n}$ for all $\left.x \in K\right)$, we will say that $b(x)$ is weakly gradient-like if for every $\epsilon>0$, there are functions $c(x)$ and $G(x)$ defined on an open neighborhood $W$ of $K$ such that:

(i) $c(x)$ is continuous on $W$ and $G(x)$ is continuously differentiable on $W$;

(ii) $c(x)>0$ on $W$;

(iii) $|c(x) \nabla G(x)-b(x)|<\epsilon$ for all $x \in K$.

In other words, a continuous vector field on $K$ is weakly gradient-like if it may be approximated uniformly on $K$ by vector fields with continuous, strictly positive integrating factors. 
THEOREM 3.2. If $f_{0}$ satisfies local growth condition (*) on $A_{0}$ and if every continuous vector field defined on $A_{0}$ is weakly gradient-like, then $I \Phi$ dominates $L$ for some $\Phi$.

Proof. By Lemma 3.1, there is a continuous vector field $b(x)$ defined in a neighborhood $W$ of $A_{0}$ and a scalar $\nu>0$ such that

$$
f_{0}\left(x, x^{\prime}\right) \geqslant b(x) \cdot x^{\prime}+\nu\left|x^{\prime}\right|
$$

for all $x$ in $W \cap A$, and $x^{\prime}$ in $Q(x)$. By assumption, given $\delta>0$, there is a continuously differentiable real-valued function $G_{0}(x)$ and a continuous, strictly positive function $c(x)$, both defined in an open neighborhood $Y$ of $A_{0}$ such that

$$
\left|c(x) \nabla G_{0}(x)-b(x)\right|<\delta
$$

for all $x$ in $A_{0}$. In particular, we may choose $\delta=\nu$, where $\nu$ appears in equation (8), so that $\left|c(x) \nabla G_{0}(x)-b(x)\right|<\nu$ for all $x$ in $A_{0}$. It is clear that for $0<\epsilon$ $<\nu$, we may find a neighborhood $X$ of $A_{0}$ such that cl $X \subseteq W \cap Y$ and $\left|c(x) \nabla G_{0}(x)-b(x)\right| \leqslant \epsilon$ for $x$ in $\mathrm{cl} X$. This implies that

$$
\left|\left(c(x) \nabla G_{0}(x)-b(x)\right) \cdot x^{\prime}\right| \leqslant \epsilon\left|x^{\prime}\right|
$$

for $x$ in $\operatorname{cl} X \cap A$ and $x^{\prime}$ in $Q(x)$, which gives

$$
c(x) \nabla G_{0}(x) \cdot x^{\prime} \leqslant b(x) \cdot x^{\prime}+\epsilon\left|x^{\prime}\right|
$$

or,

$$
c(x) \nabla G_{0}(x) \cdot x^{\prime}+(\nu-\epsilon)\left|x^{\prime}\right| \leqslant b(x) \cdot x^{\prime}+\nu\left|x^{\prime}\right|
$$

for $x$ in $\operatorname{cl} X \cap A, x^{\prime}$ in $Q(x)$. By (8), we have

$$
f_{0}\left(x, x^{\prime}\right) \geqslant c(x) \nabla G_{0}(x) \cdot x^{\prime}+(\nu-\epsilon)\left|x^{\prime}\right|
$$

for all $x$ in $\operatorname{cl} X \cap A$ and $x^{\prime}$ in $Q(x)$. Since $c(x) \geqslant c_{\min }>0$,

$$
(1 / c(x)) f_{0}\left(x, x^{\prime}\right) \geqslant \nabla G_{0}(x) \cdot x^{\prime}+[(\nu-\epsilon) / c(x)]\left|x^{\prime}\right|,
$$

for $x$ in $\operatorname{cl} X \cap A, x^{\prime}$ in $Q(x)$. Now, $1 / c(x) \leqslant 1 / c_{\min }$, so that $(1 / c(x)) f_{0}\left(x, x^{\prime}\right)$ $\leqslant\left(1 / c_{\min }\right) f_{0}\left(x, x^{\prime}\right)$. Similarly, $1 / c(x) \geqslant 1 / c_{\max }$ so that $[(\nu-\epsilon) / c(x)]\left|x^{\prime}\right| \geqslant$ $\left[(\nu-\epsilon) / c_{\max }\right]\left|x^{\prime}\right|$. Substituting in (9), we have

$$
\left(1 / c_{\min }\right) f_{0}\left(x, x^{\prime}\right) \geqslant \nabla G_{0}(x) \cdot x^{\prime}+\left[(\nu-\epsilon) / c_{\max }\right]\left|x^{\prime}\right|
$$

for $x$ in $\operatorname{cl} X \cap A, x^{\prime}$ in $Q(x)$. We let $G(x)=c_{\min } G_{0}(x)$ and $\nu^{\prime}=$ $\left(c_{\min } / c_{\max }\right)(\nu-\epsilon)$ so that $\nu^{\prime}>0$. From (10) we have

$$
f_{0}\left(x, x^{\prime}\right) \geqslant \nabla G(x) \cdot x^{\prime}+\nu^{\prime}\left|x^{\prime}\right|
$$

for $x$ in $\operatorname{cl} X \cap A, x^{\prime}$ in $Q(x)$. The hypotheses of Theorem 2.3 are thus satisfied 
by $G(x)$ defined on the open set $X$, and we conclude that $I \Phi$-dominates $L$, by Theorem 2.3.

The approximation problem thus posed by Theorem 3.2 is considered in [7]. Sufficient conditions are given in [7] for the problem when $n=2$ and when $n>2$. When the dimension $n$ is 2 , we have the following:

THEOREM 3.3. If $K$ is a compact subset of $E^{2}$ such that $K$ is nowhere dense and has connected complement in $E^{2}$, then every continuous vector field $b=\left(b_{1}, b_{2}\right)$ on $K$ is weakly gradient-like. (See [7].)

We summarize our results for $n=2$ in the following:

Corollary 3.2. If $f_{0}$ satisfies local growth condition (*) on $A_{0}$ and if $A_{0}$ is nowhere dense and has connected complement in $E^{2}$, then $I \Phi$-dominates $L$ for some $\Phi$.

As a general example of a function $f_{0}$ satisfying the conditions of Corollary 3.2, we consider the following: let $S$ be any compact subset of $E^{2}$ which is nowhere dense and has connected complement. Let $f(x, y)=\left(f_{1}(x, y), f_{2}(x, y)\right)$ be a continuous $E^{2}$-valued function on $E^{2}$ such that $|f(x, y)|=1$ for all $(x, y)$ in $S$. We define $d(x, y)=\min _{(\bar{x}, \bar{y}) \in S}\left[(x-\bar{x})^{2}+(y-\bar{y})^{2}\right]^{1 / 2}$ so that $d(x, y)$ is a continuous real-valued function on $E^{2}$. It is clear that the function $f_{0}$ defined below satisfies the hypotheses of Corollary 3.2 :

$$
\begin{aligned}
f_{0}(x, y, u, v)= & d(x, y)\left[u^{2}+v^{2}\right]^{1 / 2}+\left|u-f_{1}(x, y)\left[u^{2}+v^{2}\right]^{1 / 2}\right| \\
& +\left|v-f_{2}(x, y)\left[u^{2}+v^{2}\right]^{1 / 2}\right| .
\end{aligned}
$$

As a special case of Corollary 3.2 , we have the following results:

COROLlaRY 3.3. If $Q(x)=E^{2}$, if $f_{0}$ satisfies local growth condition (*) on $A_{0}$ and $A_{0}$ is the union of finitely many points in $E^{2}$, then I $\Phi$-dominates $L$ for some $\Phi$.

An analogue to Corollary 3.3 was first proved by Tonelli under additional smoothness assumptions on $f_{0}$ [13, Volume II, p. 12].

COROLlary 3.4. If $Q(x)=E^{2}$; if $f_{0}$ satisfies local growth condition (*) on $A_{0}$ and $A_{0}$ is the union of finitely many twice continuously differentiable arcs forming no closed curve, then $I \Phi$-dominates $L$ for some $\Phi$.

An analogue to Corollary 3.4 was first proved by Tonelli under additional smoothness assumptions on $f_{0}$ [13, Volume II, p. 26].

For $n>2$, we have results from [7] which are linked with the concepts of topological dimension (see [8]). We shall state the definitions and theorems from dimension theory that we shall need here.

Following [8, p. 24], we define the dimension of a set $K$ inductively: the 
empty set is the only set with dimension -1 : we say that $K$ has dimension $\leqslant N$ at a point $p$ in $K$ if $p$ has arbitrarily small neighborhoods whose boundaries have dimension $\leqslant N-1$; we say that $K$ has dimension $\leqslant N$ (and write $\operatorname{dim} K \leqslant N$ ) if $K$ has dimension $\leqslant N$ at each of its points; we say that $\operatorname{dim} K=N$ if $\operatorname{dim} K \leqslant N$ is true but $\operatorname{dim} K \leqslant N-1$ is false.

Let $\left\{U_{1}, U_{2}, \ldots, U_{k}\right\}$ be a covering of $K$ by open sets. We define the order of the covering $\left\{U_{1}, \ldots, U_{k}\right\}$ to be the largest integer $N$ such that there are $N+1$ members $\left\{U_{i_{1}}, \ldots, U_{i_{N+1}}\right\}$ of the covering which satisfy $\bigcap_{j=1}^{N+1} U_{i_{j}} \neq \varnothing$. If $\left\{V_{1}, \ldots, V_{l}\right\}$ is a covering of $K$ by open sets, we will say that $\left\{V_{1}, \ldots, V_{l}\right\}$ is a refinement of $\left\{U_{1}, \ldots, U_{k}\right\}$ if for each $V_{j}, j=1$, $\ldots, l$, there is an $i, 1 \leqslant i \leqslant k$, such that $V_{j} \subseteq U_{i}$. (See [8,pp. 52-53].)

By [8, p. 67], we may characterize sets $K$ satisfying $\operatorname{dim} K<N$. Namely, $\operatorname{dim} K \leqslant N$ if and only if every covering of $K$ by finitely many open sets has a refinement of order $\leqslant N$. In particular, if $\operatorname{dim} K \leqslant 0$, every covering of $K$ by finitely many open sets has a refinement $\left\{V_{1}, \ldots, V_{l}\right\}$ such that $K \subseteq \bigcup_{i=1}^{l} V_{i}$ and $V_{i} \cap V_{j}=\varnothing$ for $i \neq j$. The following theorem is then proved in [7].

THEOREM 3.4. If $K$ is a compact subset of $E^{n}$ such that $\operatorname{dim} K \leqslant 0$, then every continuous vector field $b(x)=\left(b_{1}, b_{2}, \ldots, b_{n}\right)$ on $K$ is weakly gradientlike.

The second result for $n>2$ concerns a subclass of the compact sets satisfying $\operatorname{dim} K \leqslant 1$.

We note that in Theorem 3.3, we have already stated a theorem which can be used to derive a statement of this type. For, by $\left[8\right.$, p. 41], $\operatorname{dim} E^{n}=n$ for all $n$, and, if $K \subseteq E^{n}$, then, by [8, p. 44], $\operatorname{dim} K=n$ if and only if $K$ contains a nonempty subset which is open in $E^{n}$. In particular, for $n=2, K \subseteq E^{2}$ is nowhere dense in $E^{2}$ if and only if $\operatorname{dim} K \leqslant 1$. Thus, we may restate Theorem 3.3 to say: if $K$ is a compact subset of $E^{2}$ such that $\operatorname{dim} K \leqslant 1$ and $K$ has connected complement in $E^{2}$, then every continuous vector field $b(x)=\left(b_{1}, b_{2}\right)$ on $K$ is weakly gradient-like.

We now state some definitions. We assume that $B_{1}, \ldots, B_{k}$ are open balls in the Euclidean space $E^{n}$ with centers $x_{1}, \ldots, x_{k}$, respectively. If $B_{i} \cap$ $B_{j} \neq \varnothing$ for $i \neq j$, we denote by $L_{i j}$ the undirected line segment joining $x_{i}$ and $x_{j}$. Thus, $L_{i j}=L_{j i}$. If $B_{i} \cap B_{j}=\varnothing$, we let $L_{i j}=\varnothing$ and we set $L_{i l}=\left\{x_{i}\right\}$ for each i. We let $N\left(B_{1}, B_{2}, \ldots, B_{k}\right)=\bigcup_{L_{i j}} \subseteq E^{n}$, where the union is taken for $i$, $j=1,2, \ldots, k$. We note that $N\left(B_{1}, \ldots, B_{k}\right)$ is a finite union of piecewise linear curves. If $K$ is a compact subset of $E^{n}$, we will say that $K$ has the simple chain covering property if for every $\epsilon>0$, there is a covering of $K$ by open balls $B_{1}, \ldots, B_{k}$ in $E^{n}$, each of radius less than $\epsilon$, such that $N\left(B_{1}, \ldots, B_{k}\right)$ con- 
tains no simple closed curve. It is an easy consequence of the definition that if $K$ has the simple chain covering property, then $\operatorname{dim} K<1$. The following theorem is proved in [7]:

THEOREM 3.5. If $K$ is a compact subset of $E^{n}$ satisfying the simple chain covering property, then every continuous vector field $b(x)=\left(b_{1}, \ldots, b_{n}\right)$ on $K$ is weakly gradient-like.

We summarize our results for $n>2$ in the following corollary:

COROLlaRY 3.5. If $f_{0}$ satisfies local growth condition (*) on $A_{0}$ and if $\operatorname{dim} A_{0} \leqslant 0$ or if $A_{0}$ satisfies the simple chain covering property, then $I \Phi$ dominates $L$ for some $\Phi$.

With reference to the parametric calculus of variations problem formulated in $\S 1$, we now summarize our findings.

THEOREM 3.6. Suppose that: $A$ is compact; $B$ is closed; $Q_{1}(x)$ satisfies property $(Q)$ for every $x$ in $A ; f_{0}\left(x, x^{\prime}\right) \geqslant 0$ for every $\left(x, x^{\prime}\right) \in M ; f_{0}$ satisfies local growth condition (*) on $A_{0} ; \operatorname{dim} A_{0} \leqslant 0$ or $A_{0}$ satisfies the simple chain covering property (if $n=2, A_{0}$ is nowhere dense and has connected complement suffices); and $f_{0}\left(x, x^{\prime}\right)$ is convex in the variable $x^{\prime}$ for each $x$ in $A$. Then, if $\Omega$ is nonempty, $I[\cdot]$ attains its absolute minimum.

Appendix 1. We begin by summarizing some salient facts known about parametric curves. For background material on parametric curves, we refer the reader to [3], [5] and [6].

If $h$ is a continuous map from an interval $[a, b]$ onto an interval $[c, d]$, we will say that $h$ is orientation-preserving if $h(a)=c, h(b)=d$ and $h$ is monotone nondecreasing. Suppose $x=x(u), a \leqslant u \leqslant b$, and $y=y(v), c<v<d$, are A.C. (absolutely continuous) functions with values in $E^{n}$. If, given $\epsilon>0$, there is an orientation-preserving homeomorphism $h_{e}$ mapping $[a, b]$ onto $[c, d]$ such that $\left|x(u)-y\left(h_{\epsilon}(u)\right)\right|<\epsilon$ for all $u$ in $[a, b]$, then $x$ and $y$ are said to be Fréchet equivalent. In this case, we will write $x \sim y$. That the relation $\sim$ is an equivalence relation has been shown in $[3$, p. 494], for instance. A parametric curve, or Fréchet curve, is an equivalence class under $\sim$, and an A.C. function $\boldsymbol{x}$ is said to be a parametric representation for the Fréchet curve determined by $x$. (We note that the usual definitions and theorems are given in terms of continuous parametric representations. However, we will need to consider only A.C. parametric representations and hence, for simplicity, we restrict our discussion accordingly.)

THEOREM A1.1. If $x=x(u), a \leqslant u \leqslant b$, is A.C. with values in $E^{n}$, there is a unique A.C. $E^{n}$-valued function $X=X(s), 0 \leqslant s \leqslant L(x)$, and an A.C. 
orientation-preserving function $s=s(u), a \leqslant u \leqslant b$, such that: $X \sim x ;\left|X^{\prime}(s)\right|$ $=1$ almost everywhere in $[0, L(x)]$ and $X(s(u))=x(u)$ for all $u$ in $[a, b][3$, pp. 497-498].

The function $X$ is called the arc-length representation for the curve determined by $x$. Since $X(s(u))=x(u)$, we may conclude that $X^{\prime}(s(u)) s^{\prime}(u)=$ $x^{\prime}(u)$ for almost all $u$, provided the following convention is observed: $X^{\prime}(s(u)) s^{\prime}(u)$ $=0$ for all $u$ such that $s^{\prime}(u)=0$ (whether or not $X^{\prime}(s(u)$ ) is defined at such points $u$ ). We will now show that properties (2)-(5) of $\S 1$ are invariant under Fréchet equivalence, assuming the set $Q(x)$ is a cone for each $x$ in $A$.

ThEOREM A1.2. Suppose that $x=x(u), a \leqslant u \leqslant b$, and $y=y(v), c \leqslant$ $v \leqslant d$, are A.C. functions with values in $E^{n}$, and that for each $x$ in $A, Q(x)$ is a cone. If $x \sim y$, then $x$ is in $\Omega$ if and only if $y$ is in $\Omega$.

Proof. From Theorem A1.1, it suffices to prove the theorem in case there is an orientation-preserving A.C. function $u=u(v)$ mapping $[c, d]$ onto $[a, b]$ such that $x(u(v))=y(v)$. In this case, it follows that $x(u) \in A$ if and only if $y(v) \in A$. Also, $(x(a), x(b)) \in B$ if and only if $(y(c), y(d)) \in B$. Further, it is well known that $f_{0}\left(x(u), x^{\prime}(u)\right)$ is integrable if and only if $f_{0}\left(y(v), y^{\prime}(v)\right)$ is integrable, the respective integrals having the same value. Now, by assumption, $u^{\prime}(v) \geqslant 0$ for almost all $v$.

Since $x \in \Omega, x^{\prime}(u) \in Q(x(u))$ for a.a. $u$. Let $V_{1}$ be the set of $v$ in $[c, d]$ such that $x^{\prime}(u(v))$ exists and $x^{\prime}(u(v)) \in Q(x(u(v)))$. Then, $u\left(V_{1}\right)$ has measure $b-a$, and if $V_{2}=[c, d] \backslash V_{1}$, then $u\left(V_{2}\right)=[a, b] \backslash u\left(V_{1}\right)$ and $u\left(V_{2}\right)$ has measure zero. As a consequence of the fact that $u\left({ }^{\circ}\right)$ is A.C., then, $u^{\prime}(v)=0$ for a.a. $v$ in $V_{2}$. Let $V_{3}$ be the set of $v$ in $V_{1}$ such that $u^{\prime}(v)$ exists and is nonnegative, and such that $y^{\prime}(v)=x^{\prime}(u(v)) u^{\prime}(v)$. Thus, the measure of $V_{1} \backslash V_{3}$ is zero. For $v$ in $V_{3}, x^{\prime}(u(v)) \in Q(x(u(v)))=Q(y(v))$, so that $x^{\prime}(u(v)) u^{\prime}(v) \in$ $Q(y(v))$ by the cone property of $Q(\cdot)$. Therefore, $y^{\prime}(v) \in Q(y(v))$ for a.a. $v$ in $V_{1}$. Since $u^{\prime}(v)=0$ for a.a. $v$ in $V_{2}, y^{\prime}(v)=0$ for a.a. $v$ in $V_{2}$. But $0 \in Q(v(v))$ by assumption, so that $y^{\prime}(v) \in Q(y(v))$ for a.a. $v$ in $V_{2}$. We conclude that $y^{\prime}(v)$ $\in Q(y(v))$ for a.a. $v$ in $[c, d]$, so that $y \in \Omega$.

We now assume that $y \in \Omega$, so that $y^{\prime}(v) \in Q(y(v))=Q(x(u(v)))$ for a.a. $v$ in $[c, d]$. We may rewrite this relation as $x^{\prime}(u(v)) u^{\prime}(v) \in Q(x(u(v)))$ for a.a. $v$.

Let:

$$
\begin{aligned}
& V_{1}=\left\{v \in[c, d] \mid x^{\prime}(u(v)) \text { exists }\right\} \\
& V_{2}=\left\{v \in[c, d] \mid u^{\prime}(v) \text { exists, } u^{\prime}(v)>0\right\}, \\
& V_{3}=\left\{v \in[c, d] \mid x^{\prime}(u(v)) u^{\prime}(v) \in Q(x(u(v)))\right\}, \\
& V_{4}=[c, d] \backslash\left(V_{1} \cap V_{2} \cap V_{3}\right) .
\end{aligned}
$$


We have already seen that $u\left(V_{1}\right)$ has measure $b-a$. Since $u^{\prime}(v)=0$ for a.a. $v$ in $[c, d] \backslash V_{2}, u\left(V_{2}\right)$ also has measure $b-a$. Since $[c, d] \backslash V_{3}$ has measure zero and $u$ is A.C., $u\left([c, d] \backslash V_{3}\right)$ has zero measure, and $u\left(V_{3}\right)$ has measure $b-a$. Therefore, $u\left(V_{4}\right)$ has zero measure. For $v$ in $V_{1} \cap V_{2} \cap V_{3}$, then, $x^{\prime}(u(v)) u^{\prime}(v)$ $\in Q(x(u(v)))$ and $u^{\prime}(v)>0$ so that $x^{\prime}(u(v)) \in Q(x(u(v)))$ for all $u(v)$ such that $v \in V_{1} \cap V_{2} \cap V_{3}$. Therefore, $x^{\prime}(u) \in Q(x(u))$ for all $u \in u\left(V_{1} \cap V_{2} \cap V_{3}\right)$, i.e., for a.a. $u$ in $[a, b]$. Thus, $x \in \Omega$ and Theorem A1.2 is proved.

We conclude Appendix 1 with the observation that, under the assumptions on $f_{0}$, it is well known that $I[x]=I[y]$ whenever $x \sim y$ (see $[3$, p.500], for example). Therefore, we have shown that the problem posed in $\S 1$ is a parametric problem, and we shall say that $x$ and $y$ are two representations of the same path curve in $E^{n}$. For parametric Lagrange problems of optimal control, if $x \sim y$, are Fréchet equivalent, $x=x(t), a \leqslant t \leqslant b, y=y(\tau), c \leqslant \tau \leqslant d$; and $X=X(s), 0 \leqslant s \leqslant L$, is the unique arc length representation, then we know that $x(t)=X(s(t)), y(\tau)=X(\sigma(\tau))$ for suitable monotone nondecreasing functions $s(t), \sigma(\tau)$. For the corresponding representations in terms of the control variable (formulas (1) and (2) of $\S 1$ ),

$$
\begin{aligned}
& I=\int_{a}^{b} f_{0}(t, x(t), u(t)) d t \\
& \quad d x / d t=f(t, x(t), u(t)), u(t) \in U(t, x(t)), t \in[a, b] \text { (a.e.), } \\
& I=\int_{0}^{L} f_{0}(s, X(s), w(s)) d s, \\
& \quad d X / d s=f(s, X(s), w(s)), w(s) \in U(s, X(s)), s \in[0, L] \text { (a.e.), } \\
& I=\int_{c}^{d} f_{0}(\tau, y(\tau), v(\tau)) d \tau, \\
& \quad d y / d \tau=f(\tau, y(\tau), v(\tau)), v(\tau) \in U(\tau, y(\tau)), \tau \in[c, d] \text { (a.e.), }
\end{aligned}
$$

we know that $u(t), w(s), v(\tau)$ may be obtained in terms of the McShane-Warfield theorem, and thus no simple relation can be expected between $u(t), w(s), v(\tau)$.

Appendix 2 (Proof of Theorem 1.1). Let $i=\inf _{x \in \Omega} I[x]$, so that $i \geqslant 0$. For $N=1,2, \ldots$, we may find $x_{N} \in \Omega$ such that $I\left[x_{N}\right] \leqslant i+1 / N$. In particular, $i \leqslant I\left[x_{N}\right] \leqslant i+1$ for all $N$. If $L=\max _{\alpha \in[i, i+1]} \Phi(\alpha)$, and $L_{N}=L\left(x_{N}\right)$ for each $N$, then $L_{N} \leqslant L$ for each $N$. By Theorem A1.1, for each $N$, there is an absolutely continuous function $X_{N}=X_{N}(s), 0 \leqslant s \leqslant L_{N}$, such that $X_{N} \sim x_{N}$ and $\left|X_{N}^{\prime}(s)\right|=1$ for almost all s. Further, since $X_{N} \sim x_{N}, X_{N} \in \Omega$ and $I\left[X_{N}\right]$ $=I\left[x_{N}\right]$. We extend the domain of definition of $X_{N}$ to the entire interval $[0, L]$, by setting $X_{N}(s)=X_{N}\left(L_{N}\right)$ for $L_{N} \leqslant s \leqslant L$. Since $X_{N}^{\prime}(s)=0$ for $L_{N}<$ $s \leqslant L$, the extended function is still an element of $\Omega$ and $I\left[X_{N}\right]$ is unchanged. We have thus defined an equi-Lipschitzian minimizing sequence $\left\{X_{N}\right\}$ on a fixed interval $[0, L]$. Since $X_{N}(s) \in A$ for each $N$ and $s$, and $A$ is compact, the sequence 
$\left\{X_{N}\right\}$ is an equicontinuous, uniformly bounded family of functions. By the Ascoli Theorem, there is a subsequence (which, for convenience, we take to be the original sequence $\left\{X_{N}\right\}$ ) which converges uniformly to a function $X=X(s)$, $0 \leqslant s \leqslant L$. It is clear that $X$ is Lipschitzian and hence absolutely continuous. Since $A$ and $B$ are closed, it follows that $X(s) \in A$ for $0 \leqslant s \leqslant L$ and $(X(0), X(L))$ $\in B$. We note that $X_{N}^{\prime}(s) \in Q_{1}\left(X_{N}(s)\right)$ for almost all $s$. We may then make use of property $(Q)$ of the sets $Q_{1}(x)$ and of Cesari's closure theorem [1] to see that $X^{\prime}(s) \in Q_{1}(X(s))$ for almost all $s$. Hence, $X^{\prime}(s) \in Q(X(s))$ for almost all $s$. It now remains to show that $I[X]=i$.

We observe that the sequence $\left\{X_{N}^{\prime}\right\}$ is uniformly bounded (by 1) in $L^{\infty}[0, L]$ (under the ess sup norm). By the Alaoglu Theorem, there is a $w^{*}$. convergent subsequence, which we again name $\left\{X_{N}^{\prime}\right\}$. It follows, since $[0, L]$ is a compact interval, that this subsequence is weakly convergent in $L^{1}[0, L]$. It is clear, on the other hand, that $X_{N}^{\prime}$ converges weakly $\left(L^{1}\right)$ to $X^{\prime}$. We may now use Cesari's Theorem on lower semicontinuity with respect to weak convergence [2]: since $f_{0}$ is nonnegative and $f_{0}\left(x, x^{\prime}\right)$ is convex in $x^{\prime}$ for each fixed $x$; since $X_{N}$ converges uniformly to $X$ and $X_{N}^{\prime}$ converges weakly $\left(L^{1}\right)$ to $X^{\prime}$, we may conclude that $I[X] \leqslant \liminf _{N \rightarrow \infty} I\left[X_{N}\right]<i$. But $X \in \Omega$ implies $I[X] \geqslant i$. The desired conclusion follows.

\section{BIBLIOGRAPHY}

1. L. Cesari, Existence theorems for weak and usual solutions in Lagrange problems with unilateral constraints. I, II, Trans. Amer. Math. Soc. 124 (1966), 369-412, 413-430. MR 34 \# 3392; \# 3393.

2. Closure theorems for orlentor flelds and weak convergence, Arch. Rational Mech. Anal. 55 (1974), 332-356. MR 50 \# 3081.

3. - Rectifiable curves and the Weierstrass integral, Amer. Math. Monthly 65 (1958), 485-500. MR 24 \# A1354.

4. - Seminormality and upper semicontinuity in optimal control, J. Optimization Theory and Appl. 6 (1970), 114-137. MR 42 \# 5139.

5. - Surface area, Ann. of Math. Studies, no. 35, Princeton Univ. Press, Princeton, N.J., 1956. MR 17, 596.

6. G. M. Ewing, Calculus of variations with applications, Norton, New York, 1969. MR 39 \# 3367.

7. R. Goor, Gradient approximation of vector fields, J. Approximation Theory 12 (1974), 385-395.

8. W. Hurewicz and H. Walman, Dimension theory, Princeton Math. Ser., vol. 4, Princeton Univ. Press, Princeton, N.J., 1941. MR 3, 312.

9. P. Kaiser, Existence theorems in the calculus of vartations, Ph.D. Dissertation, Unversity of Michigan, Ann Arbor, Mich., 1973.

10. E. J. McShane, Recent developments in the calculus of variations, Semicentennial addresses, Amer. Math. Soc., 1938, pp. 69-97.

11. E. J. McShane and R. B. Warfield, Jr., On Fillppov's implicit functions lemma, Proc. Amer. Math. Soc. 18 (1967), 41-47; addenda and corrigenda, ibid. 21 (1969), 496498. MR 34 \# 8399; 38 \# 6574. 
12. H. Rund, The HamiltonJacobi theory in the calculus of variations: It role in mathematics and physics, Van Nostrand, Princeton, N.J., 1966. MR 37 \# 5752.

13. L. Tonelli, Fondamenti di calcolo delle variazioni, Nicola Zanichelli, Bologna, 1921.

14. - Sugli integrali del calcolo delle variazioni in forma ordinaria, Ann. Scuola Norm. Sup. Pisa 2 (1934), $401-450$.

15. L. Turner, The direct method in the calculus of variations, Ph. D. Dissertation, Purdue University, Lafayette, Indiana, 1957.

DEPARTMENT OF MATHEMATICS, UNIVERSITY OF DELAWARE, NEWARK, DELAWARE 19711

Current address: Mathematics Department, General Motors Research Laboratories, 12 Mile and Mound Roads, Warren, Michigan 48090 